\title{
Sclerosing Pneumocytoma: A Carcinoma Mimicker
}

\author{
Licheng Song*, Peng Yan* and Guoxin Mo* \\ College of Pulmonary and Critical Care Medicine, Chinese PLA General Hospital, China \\ "Equal Contribution
}

\section{Introduction}

Pulmonary sclerosing pneumocytoma (PSP) is a rare benign neoplasm, predominantly occurring in middle-aged women. When first reported, PSP was thought to be vascular in origin and named pulmonary sclerosing hemangioma because of its high morphological similarity to cutaneous sclerosing hemangioma. Thanks to electron microscopy and immunofluorescence, it has been defined as being primitive respiratory epithelium-oriented and renamed as PSP [1].

Without significant clinical symptoms, most cases have been found upon regular medical examination. In some patients it may be accompanied by chest pain, cough, and chest tightness, reflecting lung tissue compression caused by increased lesion size. The characteristics of PSP are benign but atypical on computed tomography (CT), even with the appearance of adjacent pulmonary vascular structures, marginal pseudo-capsule signs, and halo signs. The 18F-FDG PET/ $\mathrm{CT}$ is a suitable alternative when the nature of the lesion cannot be distinguished on CT. However, a greater lesion size may have more active cell proliferation, which may lead to higher 18F-Fluorodeoxyglucose (18F-FDG) uptake, and be misread as a malignant neoplasm [2]. On the other hand, even though PSP is composed of two predominant cell types, including surface and round cells, and four major histological patterns, including hemorrhagic, papillary, solid, and sclerotic areas, the rate misdiagnosis by routine pathological examination is still high because of the sample's location and high compositional diversity. With immunohistochemical analysis, the diagnostic accuracy rate has remarkably improved. We present the case of a woman, with controversial pathological diagnosis of lung samples from computed tomography-guided fine needle aspiration (CT-FNA), finally diagnosed with PSP by left upper lobectomy.

\section{Case description}

A 36-year-old woman was accidentally found to have a nodule of mixed density, about $1.9 \times 2.6 \mathrm{~cm} \mathrm{[2]} \mathrm{in} \mathrm{size,} \mathrm{in} \mathrm{the} \mathrm{left} \mathrm{upper} \mathrm{lobe,} \mathrm{and}$ several micro-lymph nodes in the mediastinum and axilla on both sides. The patient denied a history of surgery, trauma, blood transfusion, or immunosuppressor administration. There were no specific symptoms, and pulmonary function test results were normal, along with results of interferon gamma release assay and other tuberculosis-related tests. The 1-3-beta-D-glucan and galactomannan antigen tests in blood and bronchoalveolar lavage fluid (BALF) were normal. Contrastenhanced CT showed a well-circumscribed and homogeneous solid tumor without blood supply, but with related bronchus stenosis and cutoff (Figure A). Whole-body FDG PET demonstrated increased FDG accumulation in the mass with a maximal standardized uptake value (SUV max) of 1.9 (Figure B).
After admission, CT-guided percutaneous fine needle tumor biopsy (CT-FNB) was performed. During the procedure, the tumor seemed firm upon penetration, with obvious bleeding. The pathology indicated crushed and inadequate samples with very little lung tissue, having mild chronic inflammation, and skeletal muscles. Periodic acid Schiff and acid fast staining results were negative. Two weeks later, we performed CT-FNB again and obtained more lung tissue samples. Micropapillary hyperplasia was observed at the end of the sample with alveolar epithelial cell atypical hyperplasia. Consequently, highly differentiated adenocarcinoma could not be excluded. However, as a young woman with few risk factors for malignant tumors, we thought it could be a benign lesion. We then performed immunohistochemical (IHC) staining. The results indicated positivity for thyroid transcription factor 1 (TTF-1, diffuse +), P40 (diffuse +), CK (partially deep stained and partially thin stained), and negative for CD56 (-), which supported the diagnosis of PSP.

The well-defined tumor mass about $1.8 \times 1.5 \times 1 \mathrm{~cm}$ [3] was removed by left upper lobectomy. IHC was positive for TTF- $1^{+}$, epithelial membrane antigen $\left(\mathrm{EMA}^{+}\right), \mathrm{Ki}-67(+2 \%)$, vimentin ${ }^{+}, \mathrm{CD}^{+} 8^{+}, \mathrm{CK}^{+}$, and negative for carcinoma embryonic antigen (CEA) (Figure $\mathrm{C}$ ).

\section{Discussion}

This case highlights the optimal diagnostic practice for a patient with controversy arising from radiography and pathology. Sufficient samples for multiple histo-cytological assessments would lead to high confidence pathological diagnoses.

\section{Pathological PSP characteristics and differential diagnosis with highly differentiated adenocarcinoma}

In our case, IHC results were: TTF- $1^{+}, \mathrm{CK}^{+}, \mathrm{EMA}^{+}$, vimentin ${ }^{+}$, $\mathrm{CD} 56, \mathrm{CD}^{+}, \mathrm{P}^{+} 0^{+}, \mathrm{CEA}$, and $\mathrm{Ki}-67(+2 \%)$. The first four markers indicate a high possibility of PSP, and the latter could exclude malignant tumors. Moreover, the fact that samples obtained by CT-FNA were sufficient for specific IHC differential diagnosis indicate the importance of minimally-invasive techniques in pathology.

As PSP is often misdiagnosed as well-differentiated lung adenocarcinoma, it is important to identify key differences in pathological characteristics. PSP affects more female patients and there

*Correspondence to: Guoxin Mo, College of Pulmonary and Critical Care Medicine, Chinese PLA General Hospital, No. 28 Fuxing Road, Beijing 100048, China, E-mail: moguoxin@163.com

Received: January 24, 2021; Accepted: February 20, 2021; Published: February 23,2021 


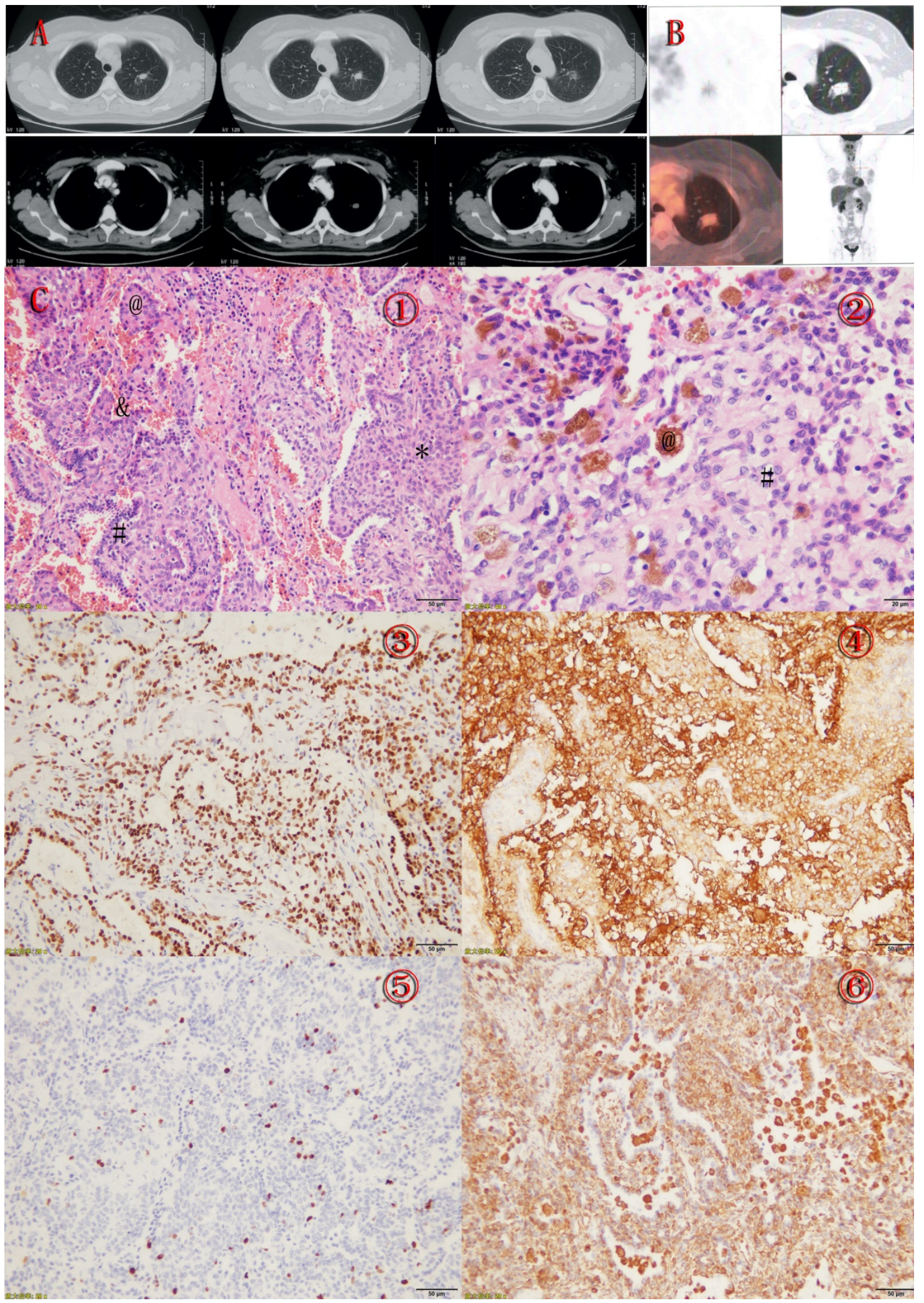

Figure A: Contrast enhanced CT showing a well-circumscribed and homogeneous solid tumor with no blood supply and related bronchus stenosis and cut off.

Figure B: PET-CT showing a solid nodule (about $1.8 \times 1.7 \mathrm{~cm}$ ) with increased FDG uptake in a 36-year-old female (SUVmax 1.9) in the left upper lobe.

Figure C: Pathological findings of pulmonary sclerosing pneumocytoma in a 36-year-old female. (1) The papillary structure (@), the typical cuboidal surface cells (\#) and stromal round cells (*) and hemorrhagic areas (\&).2)Areas of hemosiderosis (@) and solid (\#)(hematoxylin-eosin stain; original magnification, A x200 and B x400). (3) Both surface cuboidal cells and round stromal cells positive for TTF-1 (x400). (4) Both surface cuboidal cells and round stromal cells positive for EMA (x400). (5) The Ki-67 staining indicated low proliferation index (x200). (6) Vimentin stained only round cells (x200). 
is no significant correlation with risk factors such as smoking, nor hemorrhagic background or hyalinized stroma in adenocarcinoma, as well as round and surface cells, but it is characterized by widespread dyskaryotic cells and necrosis. Further, TTF-1 and Napsin-A expression patterns in adenocarcinoma are homogenous, but differ between round and surface cells in PSP [3].

\section{The significance of CT and PET-CT in PSP diagnosis and treatment}

PSP lacks typical features on CT. The halo sign, air crescent sign, air-gap-like lesion, calcification, and lobulated nodules have limited value in PSP diagnosis, while the "overlying vessel sign" and "marginal pseudo-capsule sign" on contrast-enhanced CT have a relatively higher diagnostic value [4]. For a young woman with a single nodule that is round to oval in shape, with a smooth boundary and strong and homogeneous enhancement on contrast-enhanced CT, with or without overlying vessel sign, halo sign, or air crescent sign, PSP should be considered. Nevertheless, the accuracy rate of enhanced CT on PSP diagnosis is only $30 \%$ [4].

PSP on PET-CT often shows a single nodule with relatively higher metabolism than other benign tumors such as hamartoma $[5,6]$. Furthermore, the standardized uptake value max (SUVmax) correlates significantly with the mass diameter, which leads to misdiagnosis when for diameters $>2 \mathrm{~cm}$ on PET-CT. The intense uptake may be caused by the active proliferation of variable tumor cells and poorly differentiated round cells.

\section{Need for CT-FNA}

In this case, the patient underwent several CT examinations, but PET-CT could not exclude the malignant tendency. The pathological results of samples from two CT-FNA indicated the importance of IHC, which could have helped the patient avoid lobectomy. Whether CTFNA is efficient enough for PSP diagnosis is controversial. Since it is a less invasive biopsy technique, obtaining enough tumor samples for pathology and IHC examination remains a challenge. For a 36-year old female without history of smoking or other risk factors for cancer, the long-term prognosis was not optimistic if the left upper lobe was excised. As a benign tumor, whether aggressive surgery is necessary or follow-up visits are acceptable is still controversial. Even for an undefined tumor needing resection, the determination of its invasive nature determines the surgery range and lymph node dissection. Currently, the majority of pathological PSP diagnoses are made post-surgery, so precise pathology using a fine needle may help avoid invasive surgery [7].

\section{Conclusion}

In this case report, we explored, in-depth, the diagnostic efficiency of CT and PET-CT, combined with pathological analysis, in the diagnosis of PSP. We highlighted the importance of CT-FNA in the acquisition of pathological specimens, especially for young to middleaged females with amiable imaging changes and no clinical symptoms. The differential diagnosis of PSP from adenocarcinoma and carcinoid through characteristic round and surface cells as well as specific IHC staining plays a critical role in optimizing treatment strategy.

\section{References}

1. Travis WD, Brambilla E, Nicholson AG, Yatabe Y, Austin JHM, et al. (2015) The 2015 World Health Organization Classification of Lung Tumors: Impact of Genetic, Clinica and Radiologic Advances Since the 2004 Classification. J Thorac Oncol 10: 12431260. [Crossref]

2. Cheung YC, Ng SH, Chang JW, Tan CF, Huang SF, et al. (2003) Histopathological and CT features of pulmonary sclerosing haemangiomas. Clin Radiol 58: 630-635. [Crossref]

3. Hissong E, Rao R (2017) Pneumocytoma (sclerosing hemangioma), a potential pitfall. Diagn Cytopathol 45: 744-749. [Crossref]

4. Shin SY, Kim MY, Oh SY, Lee HJ, Hong SA, et al. (2015) Pulmonary sclerosing pneumocytoma of the lung: CT characteristics in a large series of a tertiary referra center. Medicine (Baltimore) 94: e498. [Crossref]

5. Lee E, Park CM, Kang KW, Goo JM, Kim MA, et al. (2013) 18F-FDG PET/CT features of pulmonary sclerosing hemangioma. Acta Radiol 54: 24-29. [Crossref]

6. Jiang L, Huang Y, Tang Q, Zhao Q, Li Y, et al. (2018) 18F-FDG PET/CT characteristics of pulmonary sclerosing hemangioma vs. pulmonary hamartoma. Oncol Lett 16: 660665. [Crossref]

7. Dettrick A, Meikle A, Fong KM (2014) Fine-needle aspiration diagnosis of sclerosing hemangioma (pneumocytoma): report of a case and review of the literature. Diagn Cytopathol 42: 242-246. [Crossref]

Copyright: (C2021 Song L. This is an open-access article distributed under the terms of the Creative Commons Attribution License, which permits unrestricted use, distribution, and reproduction in any medium, provided the original author and source are credited. 